\title{
291.
}

\section{ON THE DEMONSTRATION OF A THEOREM RELATING TO THE MOMENTS OF INERTIA OF A SOLID BODY.}

[From the Quarterly Journal of Pure and Applied Mathematics, vol. IV. (1861), pp. 25-27.]

Considering in the first instance the analogous question in plano, let $a=\int x^{2} d m$, $b=\int y^{2} d m, h=\int x y d m$, where the integration extends over any closed figure whatever, then it is to be shown that the equations

$$
(a, h, b)(p, q)^{2}=1, \text { and }(a+b)\left(p^{2}+q^{2}\right)-(a, h, b)(p, q)^{2}=1,
$$

represent respectively ellipses.

If in the first case, by a transformation of coordinates,

$$
(a, h, b)(p, q)^{2}=a_{1} p_{1}^{2}+b_{1} q_{1}^{2},
$$

then $a_{1}, b_{1}$ are the roots of the quadratic equations,

$$
\left|\begin{array}{cc}
a-\rho, & h \\
h, & b-\rho
\end{array}\right|=0
$$

and if in the second case,

$$
(a+b)\left(p^{2}+q^{2}\right)-(a, h, b)(p, q)^{2}=a_{1} p_{1}^{2}+b_{1} q_{1}^{2},
$$

then $a_{1}, b_{1}$ are the roots of

$$
\left|\begin{array}{cc}
b-\rho, & -h \\
-h, & a-\rho
\end{array}\right|=0,
$$

the two equations being in fact the same equation,

$$
\rho^{2}-(a+b) \rho+a b-h^{2}=0,
$$


and the conditions that the curve may be an ellipse, are

$$
\begin{array}{r}
a+b=+, \\
a b-h^{2}=+,
\end{array}
$$

the former of which requires no demonstration; to prove the latter, changing merely the variables under the integral sign, I write

$$
a^{\prime}=\int x^{\prime 2} d m^{\prime}, \quad b^{\prime}=\int y^{\prime 2} d m^{\prime}, \quad h^{\prime}=\int x^{\prime} y^{\prime} d m^{\prime},
$$

these quantities being of course respectively equal to $a, b, h$, we have then

$$
a b^{\prime}+a^{\prime} b-2 h h^{\prime}=\iint\left(x y^{\prime}-x^{\prime} y\right)^{2} d m d m^{\prime}=2\left(a b-h^{2}\right),
$$

or since the quantity under the integral sign is a square, $a b-h^{2}$ is positive.

For the analogous problem in solido, we have

$$
a=\int x^{2} d m, b=\int y^{2} d m, c=\int z^{2} d m, f=\int y z d m, g=\int z x d m, h=\int x y d m,
$$

and it is to be shown that the equations

$$
\begin{gathered}
(a, b, c, f, g, h)(p, q, r)^{2}=1 \\
(a+b+c)\left(p^{2}+q^{2}+r^{2}\right)-(a, b, c, f, g, h)(p, q, r)^{2}=1
\end{gathered}
$$

represent respectively ellipsoids.

The conditions in the first problem are

$$
\begin{array}{rr}
a+b+c & =+ \\
b c+c a+a b-f^{2}-g^{2}-h^{2} & =+ \\
a b c-a f^{2}-b g^{2}-c h^{2}+2 f g h & =+
\end{array}
$$

the first of which is obviously true: as regards the second, the theorem in plano shows that each of the quantities $b c-f^{2}, c a-g^{2}, a b-h^{2}$ is positive, or merely reproducing the investigation, we find

$$
2\left(b c+c a+a b-f^{2}-g^{2}-h^{2}\right)=\iint\left[\left(y z^{\prime}-y^{\prime} z\right)^{2}+\left(z x^{\prime}-z^{\prime} x\right)^{2}+\left(x y^{\prime}-x^{\prime} y\right)^{2}\right] d m d m^{\prime},
$$

which proves the theorem, and where it is to be observed that the integral may also be written

$$
\iint\left[\left(x^{2}+y^{2}+z^{2}\right)\left(x^{\prime 2}+y^{\prime 2}+z^{\prime 2}\right)-\left(x x^{\prime}+y y^{\prime}+z z^{\prime}\right)^{2}\right] d m d m^{\prime}
$$

and for the third, we find in a precisely similar manner,

$$
6\left(a b c-a f^{2}-b g^{2}-c h^{2}+2 f g h\right)=\iint\left|\begin{array}{lll}
x, & y, & z \\
x^{\prime}, & y^{\prime}, & z^{\prime} \\
x^{\prime \prime}, & y^{\prime \prime}, & z^{\prime \prime}
\end{array}\right|^{2} d m d m^{\prime}
$$


which proves the theorem. The integral may also be written

$$
\iint\left|\begin{array}{ccc}
x^{2}+y^{2}+z^{2}, & x x^{\prime}+y y^{\prime}+z z^{\prime}, & x x^{\prime \prime}+y y^{\prime \prime}+z z^{\prime \prime} \\
x^{\prime} x+y^{\prime} y+z^{\prime} z, & x^{\prime 2}+y^{\prime 2}+z^{\prime 2}, & x^{\prime} x^{\prime \prime}+y^{\prime} y^{\prime \prime}+z^{\prime \prime} z^{\prime \prime} \\
x^{\prime \prime} x+y^{\prime \prime} y+z^{\prime \prime} z, & x^{\prime \prime} x^{\prime}+y^{\prime \prime} y^{\prime}+z^{\prime \prime} z^{\prime}, & x^{\prime \prime 2}+y^{\prime \prime 2}+z^{\prime 2}
\end{array}\right| d m d m^{\prime} .
$$

The conditions in the second problem are

$$
\begin{aligned}
& (b+c)+(c+a)+(a+b)=+, \\
& (c+a)(a+b)+(a+b)(b+c)+(b+c)(c+a)-f^{2}-g^{2}-h^{2}=+, \\
& (a+b)(b+c)(c+a)-(b+c) f^{2}-(c+a) g^{2}-(a+b) h^{2}-2 f g h=+,
\end{aligned}
$$

the first and second of which are respectively equivalent to

$$
\begin{aligned}
& a+b+c=+, \\
& (a+b+c)^{2}+b c+c a+a b-f^{2}-g^{2}-h^{2}=+,
\end{aligned}
$$

which are already proved. The last may be written

$$
(a+b+c)\left(b c+c a+a b-f^{2}-g^{2}-h^{2}\right)-\left(a b c-a f^{2}-b g^{2}-c h^{2}+2 f g h\right)=+,
$$

which, putting for shortness,

$$
\begin{aligned}
& A=x^{2}+y^{2}+z^{2}, B=x^{\prime 2}+y^{\prime 2}+z^{\prime 2}, C=x^{\prime \prime 2}+y^{\prime \prime 2}+z^{\prime \prime 2} \\
& F=x^{\prime} x^{\prime \prime}+y^{\prime} y^{\prime \prime}+z^{\prime} z^{\prime \prime}, G=x^{\prime \prime} x+y^{\prime \prime} y+z^{\prime \prime} z, H=x x^{\prime}+y y^{\prime}+z z^{\prime}
\end{aligned}
$$

is by what precedes expressible in the form

$$
\begin{aligned}
\frac{1}{6} \iint\left\{A\left(B C-F^{2}\right)+B\left(C A-G^{2}\right)\right. & \left.+C\left(A B-H^{2}\right)-\left(A B C-A F^{2}-B G^{2}-C H^{2}+2 F G H\right)\right\} d m d m^{\prime} \\
& =\frac{1}{3} \iint(A B C-F G H) d m d m^{\prime}
\end{aligned}
$$

or, since $\sqrt{B C}>F, \sqrt{C A}>G, \sqrt{A B}>H$, we have $A B C>F G H$, or $A B C-F G H=+$, and therefore the value of the integral is also positive.

2, Stone Buildings, W.C., 6th March, 1860. 